\title{
Accuracy of ICD Codes for Identification: Review of Chlamydia, Gonorrhea and Syphilis
}

\author{
Yenling A. Ho ${ }^{1}$, Saurabh Rahurkar ${ }^{2}$, Janet Arno3, 4 , Brian E. Dixon 1, 2, 5 \\ ${ }^{1}$ Indiana University Fairbanks School of Public Health, Indianapolis, Indiana, United States, ${ }^{2}$ Regenstrief Institute, Indianapolis, Indiana, United States, \\ ${ }^{3}$ Indiana University School of Medicine, Indianapolis, Indiana, United States, ${ }^{4}$ Marion County Public Health Department, Indianapolis, Indiana, United \\ States, ${ }^{5}$ Roudebush VA Medical Center, Indianapolis, Indiana, United States
}

\section{Objective}

The purpose of this study is to review the extant literature for evidence on the validity of ICD-9-CM and -10-CM codes for the purpose of identifying cases of chlamydia, gonorrhea, and syphilis.

\section{Introduction}

Administrative data refers to data generated during the processes of health care. These data are a rich source of patient health information, including diagnoses and problem lists, laboratory and diagnostic tests, and medications. Established standards are used to code each data into the appropriate coding systems. The International Classification of Diseases, Ninth and Tenth Revisions, Clinical Modification (ICD-9-CM and ICD-10-CM) codes are the coding standard for diagnoses and have been frequently used to identify cases for the creation of cohorts in examining care delivery, screening, prevalence, and risk factors [1,2].

However, while some studies have assessed the validity and reliability of ICD-CM codes to identify various conditions such as cerebral palsy and rheumatoid arthritis $[3,4]$, the evidence for using ICD codes to accurately identify sexually transmitted infection (STI) cases is largely unexamined. The purpose of this study is to review the extant literature for evidence on the validity of ICD codes for identifying cases of chlamydia, gonorrhea, and syphilis. Our findings will inform efforts to improve the use of administrative data for STI-related health service and surveillance researches.

\section{Methods}

Our systematic review followed a protocol consistent with the Preferred Reporting Items for Systematic Reviews and MetaAnalysis (PRISMA). We comprehensively searched PubMed and Scopus databases for peer-reviewed articles published before February 2018. Articles were identified with search terms related to our STIs of interest (chlamydia, syphilis or gonorrhea), pelvic inflammatory disease (PID), administrative codes, and validation studies. PID was included as 33\%-50\% of PID cases are due to chlamydia or gonorrhea [5]. Only empirical publications appearing in peer-reviewed English language journals were included.

Further, we excluded articles classified as letters to the editor, policy briefs, perspectives, commentaries, summaries of future research plans, and grey literature. Additionally, articles without abstracts were also excluded. The screening process used by our review is outlined in Figure 1. Briefly, all articles were subjected to a two-step screening process. First, we reviewed articles based on title and abstract. We eliminated studies that did not focus on STIs or on validation in the context of STIs. Articles were included if they focused on any combination of the STIs of interest, or on PID, and were validation studies on diagnostic testing or administrative codes. Second, selected articles were then reviewed in full to identify studies which included the STIs of interest, assessed and listed ICD-9-CM or -10-CM codes, and measured validity. The snowball technique was used on included articles, whereby we reviewed all references found in the references of the included articles.

\section{Results}

Our search strategy identified 1,754 articles to be screened by title and abstract. Of these, only five $(0.29 \%)$ articles met the initial inclusion criteria. After full text review, only two articles [6,7] met the final inclusion criteria to be included in the systematic review. Both articles focused on PID with no assessment of syphilis. They utilized ICD-9-CM codes to identify cases with PID and performed chart reviews to determine true PID status. Results of both articles found positive predictive value (PPV) of PID to be between 18\%-79\%. Only one article [7] examined the PPV of chlamydia (56\%; 5/9 cases) and gonorrhea (100\%; 4/4 cases) separately. 


\section{Conclusions}

We identified just two studies that evaluated the validity of ICD codes in identifying the STIs of interest. Both studies focused on PID cases in which chlamydia and gonorrhea diagnoses and tests might be documented. Additionally, since both studies were published before 2015, neither evaluates the use of administrative data following the U.S. transition to ICD-10 codes. Given these findings, further studies are required to examine the predictive value of ICD-9 and -10 codes for all three diseases in the general population.

\section{Acknowledgement}

The work described is supported by the National Library of Medicine (NLM) training grant under grand number T15LM012502. The content is solely the responsibility of the authors and does not necessarily represent the official views of the NLM.

\section{References}

1. Tao G, Zhang CX. 2008. HIV Testing of Commercially Insured Patients Diagnosed With Sexually Transmitted Diseases. Sex Transm Dis. 35(1), 43-46. PubMed https://doi.org/10.1097/OLQ.0b013e318148c35a

2. Evans HE, Mercer CH, Rait G, et al. 2009. Trends in HIV testing and recording of HIV status in the UK primary care setting: a retrospective cohort study 1995-2005. Sex Transm Infect. 85, 520-26. PubMed https://doi.org/10.1136/sti.2008.034801

3. Oskoui M, Ng P, Dorais M, et al. 2017. Accuracy of administrative claims data for cerebral palsy diagnosis: a retrospective cohort study. CMAJ Open. 5(3), E570-75. PubMed https://doi.org/10.9778/cmajo.20170013

4. Sauer BC, Teng C-C, Accortt NA, et al. 2017. Models solely using claims-based administrative data are poor predictors of rheumatoid arthritis disease activity. Arthritis Res Ther. 19(1), 86. PubMed https://doi.org/10.1186/s13075-017-1294-0

5. Haggerty CL, Ness RB. 2006. Epidemiology, pathogenesis and treatment of pelvic inflammatory disease. Expert Rev Anti Infect Ther. 4(2), 235-47. PubMed https://doi.org/10.1586/14787210.4.2.235

6. Satterwhite CL, Yu O, Raebel MA, et al. 2011. Detection of Pelvic Inflammatory Disease: Development of an Automated Case-Finding Algorithm Using Administrative Data. Infect Dis Obstet Gynecol. 2011, 428351. https://doi.org/10.1155/2011/428351.

7. Ratelle S, Yokoe D, Blejan C, et al. 2003. Predictive value of clinical diagnostic codes for the CDC case definition of pelvic inflammatory disease (PID): implications for surveillance. Sex Transm Dis. 30(11), 866-70. PubMed https://doi.org/10.1097/01.OLQ.0000087945.08303.38 

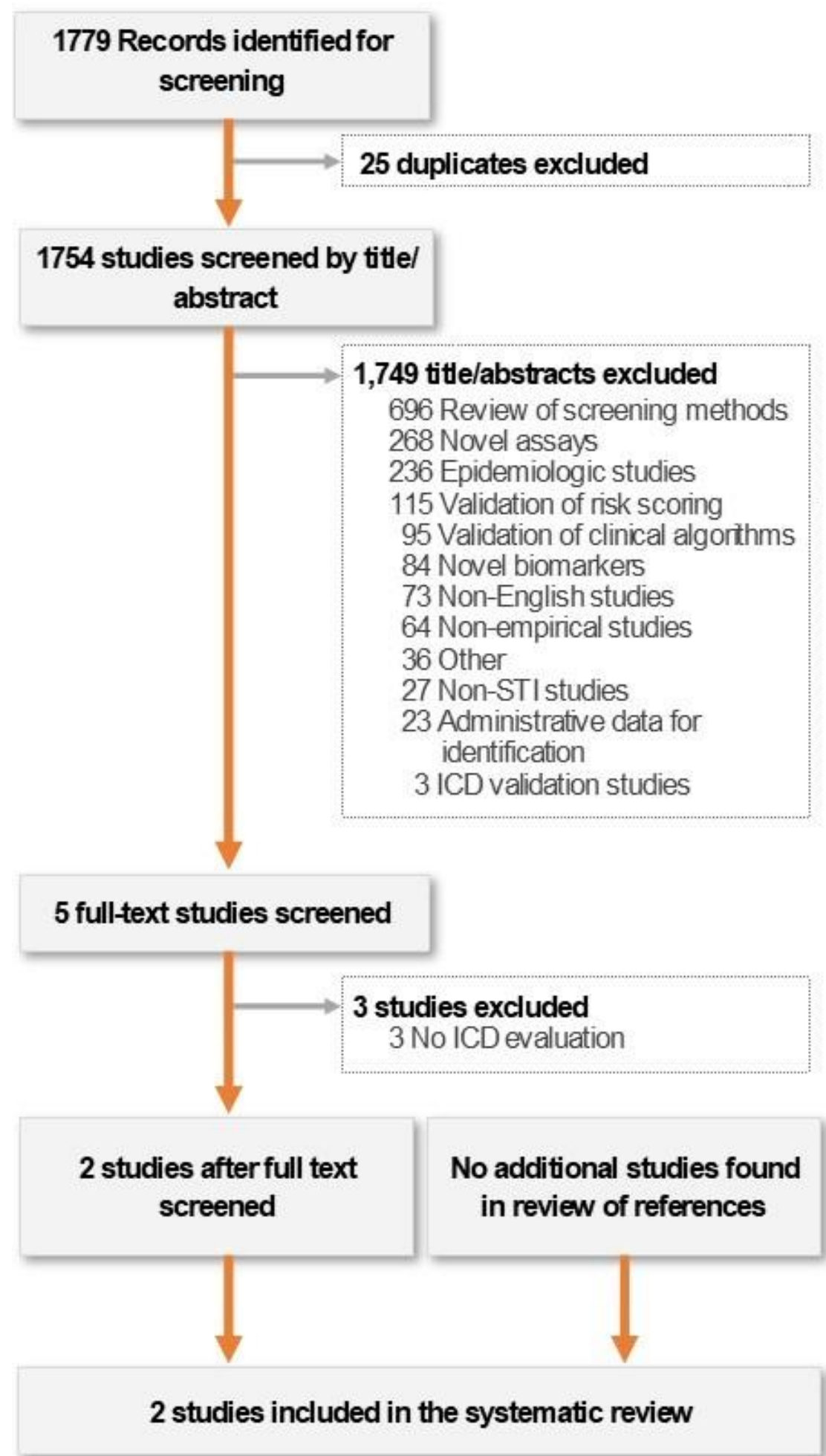

Figure 1. Flowchart of the article selection process for the systematic review. 